Published in final edited form as:

Contraception. 2019 November ; 100(5): 413-419. doi:10.1016/j.contraception.2019.07.142.

\title{
Changes in US health care provider attitudes related to contraceptive safety before and after the release of National Guidance
}

\author{
Lauren B. Zapata ${ }^{a,}{ }^{\star}$, Isabel A. Morgan ${ }^{a, b}$, Kathryn M. Curtis ${ }^{a}$, Suzanne G. Folger ${ }^{a}$, Maura K. \\ Whiteman ${ }^{\mathrm{a}}$ \\ aDivision of Reproductive Health, Centers for Disease Control and Prevention, 4770 Buford \\ Highway NE, Mailstop F-74, Chamblee, Georgia 30341-3717 \\ bOak Ridge Institute for Science and Education (ORISE), 1299 Bethel Valley Road, Oak Ridge, \\ TN 37830
}

\begin{abstract}
Objective: The US Medical Eligibility Criteria for Contraceptive Use (USMEC) is the first national guidance containing evidence-based recommendations for contraception. We describe provider attitudes about contraceptive safety before and after the 2010 USMEC release.
\end{abstract}

Study design: We conducted two cross-sectional mailed surveys using different nationwide samples of office-based physicians and Title X clinic providers before (2009-2010) and after (2013-2014) the USMEC release. We compared the proportion of providers reporting select contraceptive methods as safe for women with specific characteristics or medical conditions before and after the USMEC release and conducted multivariable logistic regression to adjust for provider characteristics.

Results: For the following select characteristics for which the USMEC classifies specific contraceptive methods as safe (Category 1 or 2), a significantly ( $\mathrm{p}<.05)$ higher proportion of providers reported the method safe after versus before the USMEC release: intrauterine devices (IUDs) for adolescents (79.8\% versus 60.2\%), IUDs for women with HIV (72.4\% versus $50.6 \%$ ), depot medroxyprogesterone acetate (DMPA) for women with obesity $(89.5 \%$ versus $76.1 \%)$, and DMPA for women with history of bariatric surgery (87.6\% versus $73.9 \%)$. These differences remained significant after adjustment for provider characteristics.

Conclusions: While we observed many positive changes in health care provider attitudes related to contraception safety after the USMEC release, gaps remain. Continuing education and evidence-based training for providers, and ensuring office and health center protocols address medical eligibility for contraception for the full range of characteristics included in the USMEC might bridge remaining gaps and increase delivery of high-quality contraception care.

*Corresponding author. Tel.: +1 770488 6358; fax: +1 770488 6391. lzapata@cdc.gov (L.B. Zapata).

Publisher's Disclaimer: Disclaimer: The findings and conclusions in this report are those of the authors and do not necessarily represent the official position of the Centers for Disease Control and Prevention or the Oak Ridge Institute for Science and Education. 
Implications: Gaps between evidence and provider attitudes remain that can inform future efforts to improve contraceptive service delivery.

\section{Introduction}

Nearly half of US pregnancies are unintended [1]. Unintended pregnancies are associated with increased risks for adverse maternal and infant health outcomes [2] and health care costs [3]. As contraceptive use is a key strategy to prevent unintended pregnancy, removing unnecessary medical barriers to accessing and using contraception may reduce the unintended pregnancy rate.

In 2010, the Centers for Disease Control and Prevention (CDC) published the U.S. Medical Eligibility Criteria for Contraceptive Use (USMEC) [4], adapted from the World Health Organization (WHO) MEC [5]. The USMEC is the first national guidance containing evidence-based recommendations for the safe use of contraception for women and men with specific characteristics and medical conditions. Updated in 2016 [6], the USMEC is intended to be a source of clinical guidance assisting health care providers when counseling about contraceptive method choice. For each included medical condition and personal characteristic, the USMEC provides recommendations for use of specific contraceptive methods, expressed as four categories. ${ }^{1}$

Health care providers can influence patient contraceptive choices during contraceptive counseling [7,8] and may limit discussion of potential methods to those perceived as suitable options. Understanding provider attitudes that may serve as medical barriers to contraception access, such as those related to safety, is important. The objective of this analysis is to describe provider attitudes about the safety of select contraceptive methods for women with specific characteristics and medical conditions before and after the release of the first USMEC.

\section{Materials and methods}

We conducted two cross-sectional mailed surveys using different nationwide samples of office-based physicians and Title X clinic providers before (Phase 1: 2009-2010) and after (Phase 2: 2013-2014) the USMEC release. Office-based physicians included specialists in obstetrics and gynecology, family medicine, and adolescent medicine, sampled from the American Medical Association Physician Masterfile. Title X clinic providers included physician and non-physician (eg, nurse, nurse practitioner, physician assistant, certified nurse midwife) clinicians working at Title X-funded health centers, sampled from structured databases maintained by the US Department of Health and Human Services Office of Population Affairs (Phase 1) or the Guttmacher Institute (Phase 2). Health centers were randomly selected by type (e.g., health department, community health center), in proportion to the relative number in the country. At each sampled health center, any eligible clinician

\footnotetext{
${ }^{1}$ A category 1 classification indicates a condition for which there is no restriction for use of the contraceptive method. A category 2 classification indicates that the advantages of using the method generally outweigh any risks, and the method generally can be used. A category 3 classification requires careful clinical judgment, and use of the method is generally not recommended unless other methods are not available or acceptable. A category 4 classification indicates that the method poses an unacceptable health risk.
} 
could complete the survey; specific clinicians were not targeted. Office-based physicians and Title $\mathrm{X}$ clinic providers were eligible to complete the survey if they provided family planning services to at least two women of reproductive age per week. Providers were asked to indicate if they considered specific contraceptive methods to be safe (very safe, safe), unsafe (very unsafe, unsafe), or if they did not know for women with specific characteristics and medical conditions. Response rates for office-based physicians were 53\% (Phase 1) and $40 \%$ (Phase 2); response rates for Title X clinic providers were 78\% (Phase 1) and 66\% (Phase 2). Other methodological details are described elsewhere $[9,10]$. The project did not require IRB approval since $\mathrm{CDC}$ determined it to be non-research, public health practice.

We combined data from both phases for office-based physicians and Title $\mathrm{X}$ clinic providers, excluding non-clinician respondents (eg, health educators, clinic administrators) ( $n=33$ ), resulting in an analytic sample of 3445 providers. We examined sample characteristics by phase to determine how respondents may have differed. For each phase, we estimated the percent of providers reporting specific methods as safe for women with select characteristics or medical conditions, overall, and by provider type and calculated the absolute difference between Phase 2 and Phase 1 estimates. We grouped USMEC classifications 1 and 2 to represent "safe" and USMEC classifications 3 and 4 to represent "unsafe". We conducted multivariable logistic regression to estimate adjusted prevalence ratios (aPRs) and 95\% confidence intervals (CIs) of the associations between phase (entered as a covariate) and provider attitudes of method safety (coded as safe versus unsafe or don't know). In modeling, we adjusted for provider characteristics that differed between phases at $\mathrm{p}<.10$ (i.e., provider type, training in IUD insertion, time since completion of formal clinical training, number of female patients of reproductive age seen per week, and proportion of female patients of reproductive age who are adolescents). Last, among Phase 2 respondents only ( $n=1460$ ), we examined provider attitudes of method safety by reported use of the USMEC in practice (defined as use of the guidance itself or any of the USMEC materials or provider tools [e.g., USMEC website, wheel, iPhone application, or color-coded summary chart]). We used SUDAAN to conduct all analyses to account for the complex sample design. We weighted data to correct for non-response and differential probability of selection into the sample by provider type to generate nationally representative estimates.

\section{Results}

The distribution of many provider characteristics was similar during Phases 1 and 2 and some characteristics differed $(\mathrm{p}<.10)$ (Table 1). Phase 2 respondents included a higher proportion of office-based physicians specializing in obstetrics and gynecology compared with Phase 1 (56.4\% versus $41.5 \%)$ and a lower proportion of office-based family medicine physicians (36.3\% vs. $51.2 \%$ ). A higher proportion of respondents in Phase 2 compared with Phase 1 completed their formal clinical training $\geq 25$ years ago (29.1\% versus $22.2 \%)$ and saw $\geq 51$ female patients of reproductive age per week (39.0\% versus $32.1 \%)$. Also, a higher proportion of providers in Phase 2 were trained in interval (i.e., not specific to postpartum) intrauterine device (IUD) insertion compared with Phase 1 (83.5\% versus 75.4\%).

Table 2 summarizes the USMEC classification and the percentage of health care providers reporting select contraceptive methods as safe for women with specific characteristics or 
medical conditions by phase. For IUDs, a significantly $(\mathrm{p}<.001)$ higher proportion of providers overall correctly reported IUDs as safe in Phase 2 compared with Phase 1 for adolescents $(78.9 \%$ versus $60.2 \%)$, postpartum women $(<10 \mathrm{~min}$ after delivery of the placenta [ $46.0 \%$ versus $28.3 \%$ ] and 10 min after delivery of the placenta to $<4$ weeks postpartum [57.9\% versus $41.1 \%]$ ), nulliparous women (94.2\% versus $75.8 \%)$, women with uterine fibroids ( $81.7 \%$ versus $65.2 \%$ ), and women with HIV (72.4\% versus $50.6 \%)$. We also observed variation in changes over time by provider type. For the safety of IUDs for nulliparous women and women with HIV, significant $(\mathrm{p}<.05)$ increases were observed for each provider type, with the greatest absolute difference detected for office-based family medicine physicians for the safety of IUDs for nulliparous women and office-based adolescent medicine physicians for the safety of IUDs for women with HIV.

Most providers (>92\%) reported depot medroxyprogesterone acetate (DMPA) as safe for adolescents in both phases and there was no significant change over time. A significantly $(\mathrm{p}<.05)$ higher proportion of providers overall correctly reported DMPA as safe in Phase 2 compared with Phase 1 for breastfeeding women ( $<1$ month postpartum [87.8\% versus $78.6 \%$ ] and $\geq 1$ month postpartum [94.9\% versus $89.4 \%])$, women with obesity $2(89.5 \%$ versus $76.1 \%$ ), women with a history of bariatric surgery ( $87.6 \%$ versus $73.9 \%$ ), and women with inflammatory bowel disease (IBD) (86.7\% versus $70.0 \%$ ) (Table 2). There was variation in changes over time by provider type: for the safety of DMPA for women with obesity, history of bariatric surgery, and IBD, significant ( $p<.05)$ increases were also observed for each provider type. Office-based family medicine physicians experienced the greatest absolute increases from Phase 1 to Phase 2 for these conditions.

For the safety of combined oral contraceptives (COCs), we examined the following characteristics and medical conditions for which COCs are classified as safe (breastfeeding women $\geq 1$ month postpartum without other risk factors for venous thrombosis [VTE] and women with obesity) or unsafe (smokers aged $\geq 35$ years and women with a history of bariatric surgery via malabsorp tive procedures). Overall, no significant changes between phases were detected except for a significant $(\mathrm{p}<.05)$ reduction in the proportion of providers incorrectly reporting COCs for women with a history of bariatric surgery via malabsorptive procedures (USMEC 3 ) as safe in Phase 1 compared with Phase 2 (63.3\% versus 53.2\%) (Table 2). We identified a few significant changes between phases by provider type.

Overall, after adjustment for provider characteristics that differed between phases, improvements $(\mathrm{p}<.05)$ in provider attitudes aligned with the USMEC were noted for most (Table 3) measures from before to after the release of the USMEC.

Among providers who participated in the Phase 2 survey (after release of the guidance), $59.2 \%$ reported use of the USMEC in prac tice. Use of the guidance varied $(\mathrm{p}<.05)$ by select provider characteristics. Specifically, use of the USMEC was highest among Title X clinic providers $(86.2 \%)$, office-based adolescent medicine physicians $(83.5 \%)$, and those who completed their formal clinical training $<5$ years ago $(89.9 \%)$; and was lowest for officebased family medicine physicians (48.4\%) and providers who completed their formal

${ }^{2}$ Body mass index $230 \mathrm{~kg} / \mathrm{m}^{2}$ 
clinical training 15-24 years ago (51.9\%) (data not shown). When asked how important certain sources were for staying informed about recommended clinical practices related to contraception, the sources most frequently reported as an important source were continuing education programs (72.8\%), professional organization publications or notifications (66.1\%), and journals (61.1\%).

A higher proportion of providers in Phase 2 who used the USMEC in practice compared with those who did not correctly reported IUDs and DMPA safe for all characteristics and medical conditions examined, although for some conditions the difference was minimal (Table 4). After adjusting for provider characteristics associated with use of the guidance, use of the USMEC was only significantly associated with provider attitudes on the safety of IUDs for immediately postpartum women ( $<10 \mathrm{~min}$ after delivery of the placenta) $(\mathrm{aPR}=1.62, \mathrm{CI}=1.18,2.21)$, IUDs for women with $\mathrm{HIV}(\mathrm{aPR}=1.30, \mathrm{CI}=1.08,1.57)$, and DMPA for women with history of bariatric surgery ( $\mathrm{aPR}=1.19, \mathrm{CI}=1.06,1.34)$. Use of the USMEC was not significantly associated with provider attitudes on the safety of COCs for any of the characteristics and medical conditions examined.

\section{Discussion}

Our cross-sectional surveys of office-based physicians and Title X clinic providers found many changes in US health care provider attitudes about the safety of contraception from before to after the initial release of the USMEC. We observed increases in the proportion of providers correctly reporting IUDs and DMPA safe for many characteristics and medical conditions. These increases remained after adjustment for provider characteristics that differed between phases.

Of attitudes examined, those related to IUD safety showed the greatest changes with absolute increases ranging from $17-22 \%$. The observation of positive changes in provider attitudes about the safety of IUDs was not surprising. Professional organizations including the American College of Obstetricians and Gynecologists and the American Academy of Pediatrics have promoted long-acting reversible contraception (LARC) as safe and effective contraceptive choices for most women through published committee opinions and policy statements [11-13]. Other professional organizations, states, and communities have also worked to promote evidence-based contraceptive practices and improve access to quality contraception services in the United States [14-18].

We observed less change related to provider attitudes about the safety of COCs, perhaps because oral contraceptives have long been a popular contraceptive method [19] and providers have greater experience prescribing them. We did find a reduction from before to after the release of the USMEC in the proportion of providers incorrectly reporting COCs as safe for women with a history of bariatric surgery via malabsorptive procedures. This condition was not in the WHO MEC, thus the USMEC provided new guidance for this condition. Nonetheless, given over half of providers incorrectly reported COCs as safe for these women after release of the guidance, efforts are needed to address this gap. This is particularly true given increasing trends in obesity among U.S. women [20] and rates of bariatric surgery [21]. Although bariatric surgical practice has evolved and the number of 
restrictive procedures conducted in recent years exceeds the number of malabsorptive procedures, nearly $20 \%$ of bariatric surgery procedures in 2017 were malabsorptive [22]. COC use among women with a history of bariatric surgery via malabsorptive procedures is classified as a USMEC 3 not because of increased risk of adverse events, but due to concerns about malabsorption of steroid hormones that could decrease contraceptive effectiveness [6].

We identified other gaps between evidence and provider attitudes that can inform future efforts to improve contraceptive service delivery. The proportion of providers in Phase 2 correctly reporting certain methods safe remains $<75 \%$ for IUDs for postpartum women and women with HIV; and for COCs for women with obesity. Further, office-based family medicine physicians often had the lowest proportion of attitudes about the safety of contraception that aligned with the USMEC, and they had the lowest use of the USMEC in practice. Increased outreach to this large segment of health care providers may improve contraception access and quality of services for women.

In our analysis, we found that slightly more than half of providers who participated in the Phase 2 survey (approximately 3 years after release of the guidance) reported using the USMEC or any related materials or provider tools. This is higher than previously found in a convenience sample of primary care and specialty physicians surveyed at national conferences, where relatively few (16\%) physicians reported using the USMEC in the first year after its release [23]. We also found differences in attitudes between users compared with non-users of the USMEC, but findings were only significant for a subset of characteristics and medical conditions examined after adjustment. This suggests that positive changes observed in provider attitudes regarding the safety of contraception may also be due to efforts of other organizations working to improve US family planning services and not attributable solely to the USMEC. Given that the USMEC is often incorporated into other sources of information, including clinical textbooks [24,25] and health center protocols, providers may be using the USMEC recommendations without knowing it. The high reported use of the USMEC among Title X clinic providers in our sample was not surprising since the federal Title $\mathrm{X}$ program incorporated the guidance into their clinic standards and protocols [26].

Our findings were subject to several limitations. Response rates were lower than desired and we were unable to determine how respondents and non-respondents differed with respect to contraceptive safety attitudes. However, we weighted the data, including for nonresponse, to be nationally representative. Provider responses were subject to social desirability error. With recent national and state efforts to promote access to LARC [14,16-18,27], it is possible that providers misreported their true attitudes about the safety of IUDs for select women. Findings related to provider attitudes on the safety of IUDs for immediately postpartum women have may limited relevance for clinicians who do not provide delivery care. Unfortunately, our survey did not assess if and how frequently respondents provided such care. Reported use of the USMEC may be underestimated. Other sources of clinical information (e.g., health center protocols) often incorporate the USMEC and providers may not realize that they are using the guidance. Our measurement of provider attitudes about method safety compared responses of safe with responses of unsafe or don't know and may 
have missed distinct nuances between provider attitudes of unsafe with lack of knowledge about safety. Last, given widespread efforts by a diverse group of stakeholders to improve contraception services the United States, positive changes observed in our analysis cannot be credited solely to the USMEC.

Although important, changes in provider attitudes related to safety do not necessarily translate to changes in practice. Behavior change is a complex process influenced by intrapersonal, interpersonal, institutional, community, and policy-level factors [28]. Choice and use of a contraceptive method by women also depends on multiple factors including patient preference, quality of care, and contraceptive method attributes (e.g., effectiveness, accessibility, acceptability). However, an analysis of Medicaid claims data indicated that clinical encounters for contraceptive management and provision of highly effective contraceptive methods increased among women with high-risk health conditions from before to after the USMEC release [29], suggesting the guidelines may influence clinical practice.

In conclusion, we observed many positive changes in provider attitudes related to the safety of contraception from before to after the release of the USMEC. However, gaps between evidence about the safety of select contraceptive methods and provider attitudes remain, as well as differences in attitudes by provider type. Continuing education and evidence-based training for providers, and ensuring office and health center protocols address medical eligibility for contraception for the full range of characteristics included in the USMEC might bridge remaining gaps and increase delivery of high-quality contraception care.

\section{Funding source:}

This project was supported in part by an appointment to the Research Participation Program at the Centers for Disease Control and Prevention administered by the Oak Ridge Institute for Science and Education through an interagency agreement between the U.S. Department of Energy and the Centers for Disease Control and Prevention.

\section{References}

[1]. Finer LB, Zolna MR. Declines in unintended pregnancy in the United States, 2008-2011. N Engl J Med 2016;374:843-52. [PubMed: 26962904]

[2]. Gipson JD, Koenig MA, Hindin MJ. The effects of unintended pregnancy on infant, child, and parental health: a review of the literature. Stud Fam Plann 2008;39:18-38. [PubMed: 18540521]

[3]. Sonfield A, Kost K, Gold RB, Finer LB. The public costs of births resulting from unintended pregnancies: national and state-level estimates. Perspect Sex Reprod Health 2011;43:94-102. [PubMed: 21651708]

[4]. U S CDC. Medical Eligibility criteria for contraceptive use, 2010. MMWR Recommendations and reports : Morbidity and mortality weekly report Recommendations and reports 2010;59:1-86.

[5]. Medical Eligibility WHO. Criteria for contraceptive use: A WHO family planning cornerstone. Geneva: World Health Organization; 2010.

[6]. Curtis KM, Tepper NK, Jatlaoui TC, et al. U.S. Medical Eligibility criteria for contraceptive use, 2016. MMWR Recommendations and reports : Morbidity and mortality weekly report Recommendations and reports. 2016;65:1-103.

[7]. Dehlendorf C, Grumbach K, Schmittdiel JA, Steinauer J. Shared decision making in contraceptive counseling. Contraception 2017;95:452-5. [PubMed: 28069491]

[8]. Minnis AM, Mavedzenge SN, Luecke E, Dehlendorf C. Provider counseling to young women seeking family planning services. Perspect Sex Reprod Health 2014;46:223-31. [PubMed: 24786186] 
[9]. Simmons KB, Zapata L, Curtis KM. Health care provider perceptions of the safety of IUDs for women with HIV. Perspect Sex Reprod Health 2018;50:67-73. [PubMed: 29603597]

[10]. Tyler CP, Whiteman MK, Zapata LB, Curtis KM, Hillis SD, Marchbanks PA. Health care provider attitudes and practices related to intrauterine devices for nulliparous women. Obstet Gynecol 2012;119:762-71. [PubMed: 22433340]

[11]. ACOG. ACOG Committee opinion no. 450: increasing use of contraceptive implants and intrauterine devices to reduce unintended pregnancy. Obstet Gynecol 2009;114:1434-8. [PubMed: 20134301]

[12]. ACOG ACOG. Committee opinion no. 735 summary: adolescents and long-acting reversible contraception: implants and intrauterine devices. Obstet Gynecol 2018;131:947-8. [PubMed: 29683905]

[13]. AAP. Contraception for adolescents. Pediatrics 2014;134:e1244-56. [PubMed: 25266430]

[14]. DeSisto CL, Estrich C, Kroelinger CD, et al. Using a multi-state learning community as an implementation strategy for immediate postpartum long-acting reversible contraception. Implementation science : IS, 12, 2017 p. 138. [PubMed: 29162140]

[15]. Kroelinger CD, Romero L, Lathrop E, et al. Meeting summary: state and local implementation strategies for increasing access to contraception during Zika preparedness and response - United States, September 2016. MMWR Morb Mortal Wkly Rep 2017;66:1230-5. [PubMed: 29121000]

[16]. Mueller T, Tevendale HD, Fuller TR, et al. Teen pregnancy prevention: implementation of a multicomponent, community-wide approach. J Adolesc Health 2017;60:S9-s17. [PubMed: 28235440]

[17]. Peipert JF, Madden T, Allsworth JE, Secura GM. Preventing unintended pregnancies by providing no-cost contraception. Obstet Gynecol 2012;120:1291-7. [PubMed: 23168752]

[18]. Ricketts S, Klingler G, Schwalberg R. Game change in Colorado: widespread use of long-acting reversible contraceptives and rapid decline in births among young, low-income women. Perspect Sex Reprod Health 2014;46:125-32. [PubMed: 24961366]

[19]. Daniels K, Mosher WD. Contraceptive methods women have ever used: United States. Natl Health Stat Rep 1982-2010;2013:1-15.

[20]. Flegal KM, Kruszon-Moran D, Carroll MD, Fryar CD, Ogden CL. Trends in obesity among adults in the United States, 2005 to 2014. JAMA 2016;315:2284-91. [PubMed: 27272580]

[21]. Johnson EE, Simpson AN, Harvey JB, Lockett MA, Byrne KT, Simpson KN. Trends in bariatric surgery, 2002-2012: do changes parallel the obesity trend? Surgery for obesity and related diseases : official journal of the American Society for Bariatric Surgery 2016;12:398-404. [PubMed: 26707931]

[22]. American Society for Metabolic and Bariatric Surgery. Estimate of bariatric surgery numbers, 2018, 2011-2017.

[23]. Russo JA, Chen BA, Creinin MD. Primary care physician familiarity with U.S. medical eligibility for contraceptive use. Fam Med 2015;47:15-21. [PubMed: 25646873]

[24]. Hatcher RA, Nelson AL, Trussell J, et al. Contraceptive technology 21st ed. New York, NY: Ayer Company Publishers, Inc; 2018.

[25]. Hatcher RA, Zieman M, Allen AZ, Lathrop E, Haddad L. Managing contraception 2017-2018. 14th ed. Bridging the Gap Foundation: Tiger, GA; 2017.

[26]. Gavin L, Moskosky S, Carter M, et al. Providing quality family planning services: recommendations of CDC and the U.S. Office of Population Affairs. MMWR Recommendations and reports : Morbidity and mortality weekly report Recommendations and reports. 2014;63:154.

[27]. Kroelinger CD, Morgan IA, DeSisto CL, et al. State-identified implementation strategies to increase uptake of immediate postpartum long-acting reversible contraception policies. J Womens Health (Larchmt) 2019;28(3):346-56. [PubMed: 30388052]

[28]. National Cancer Institute. Theory at a glance: A guide for health promotion practice 2nd ed:. U.S. Department of Health and Human Services National Institutes of Health; 2005.

[29]. Pujol TA, Serban N, Swann J, Kottke M. Medicaid claims for contraception among women with Medical conditions after release of the US Medical Eligibility criteria for contraceptive use. Prev Chronic Dis 2019;16:E03. [PubMed: 30605421] 


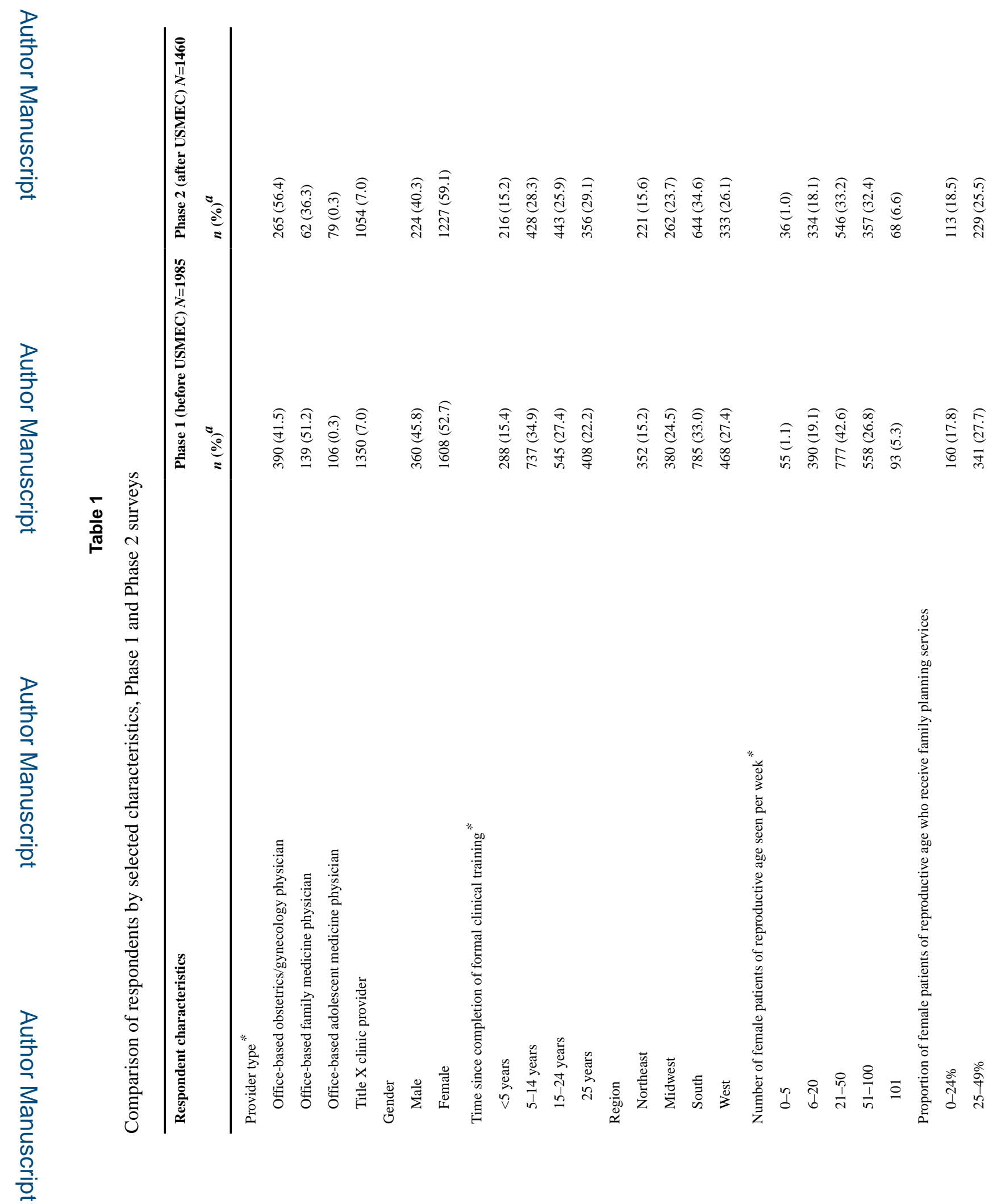

Contraception. Author manuscript; available in PMC 2020 July 22. 
Zapata et al.

Page 10

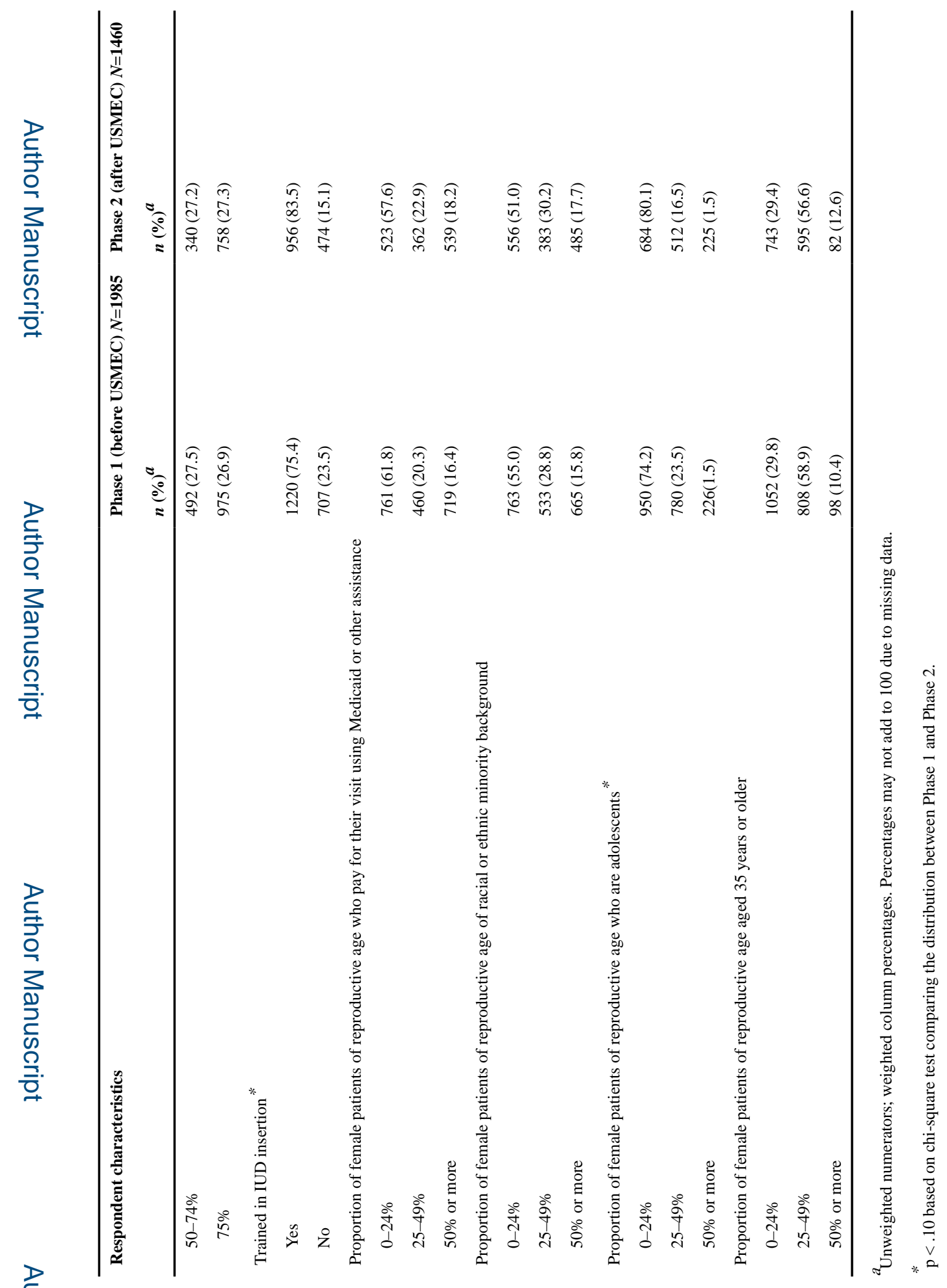

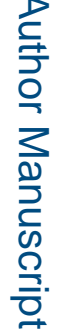

Contraception. Author manuscript; available in PMC 2020 July 22. 


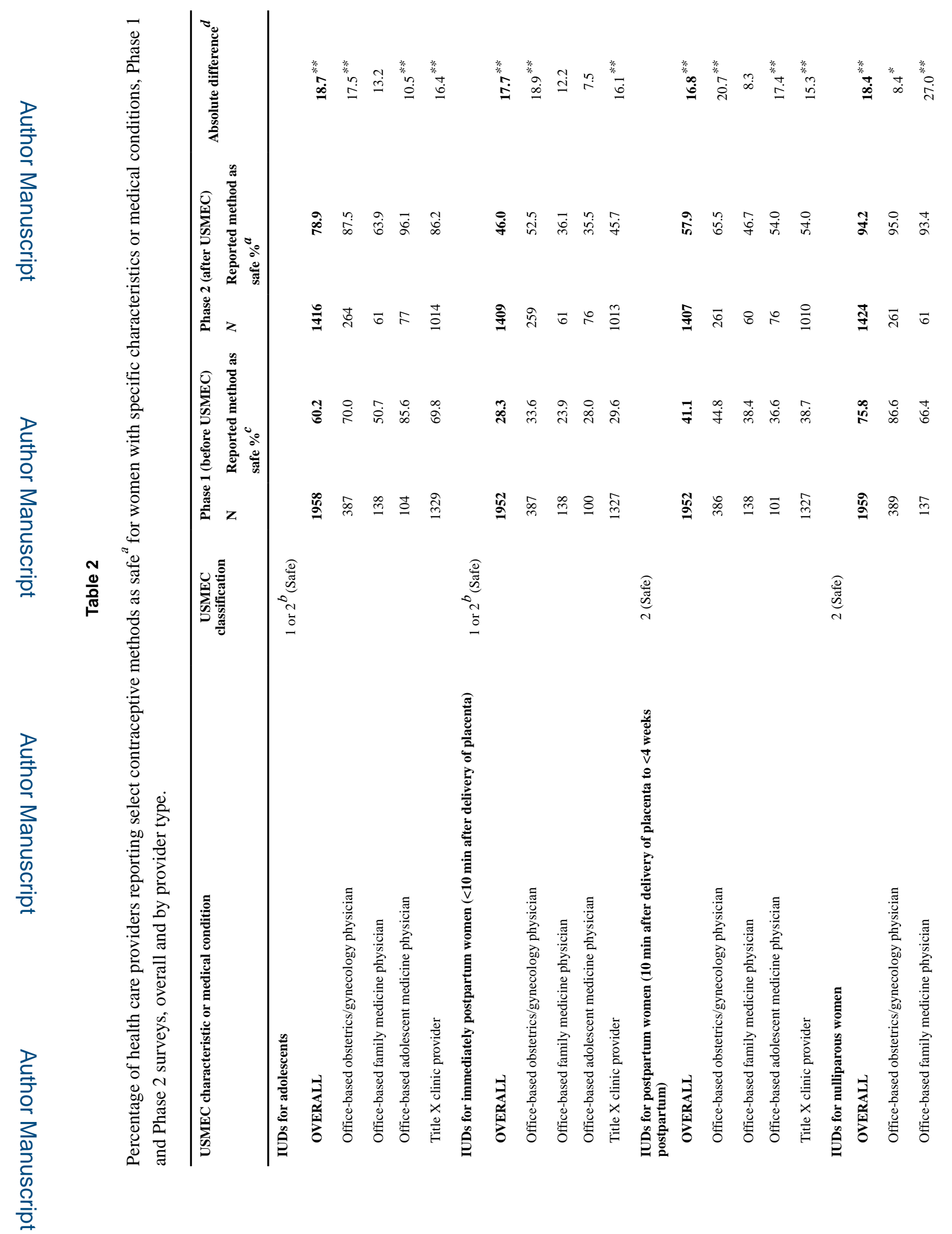

Contraception. Author manuscript; available in PMC 2020 July 22. 


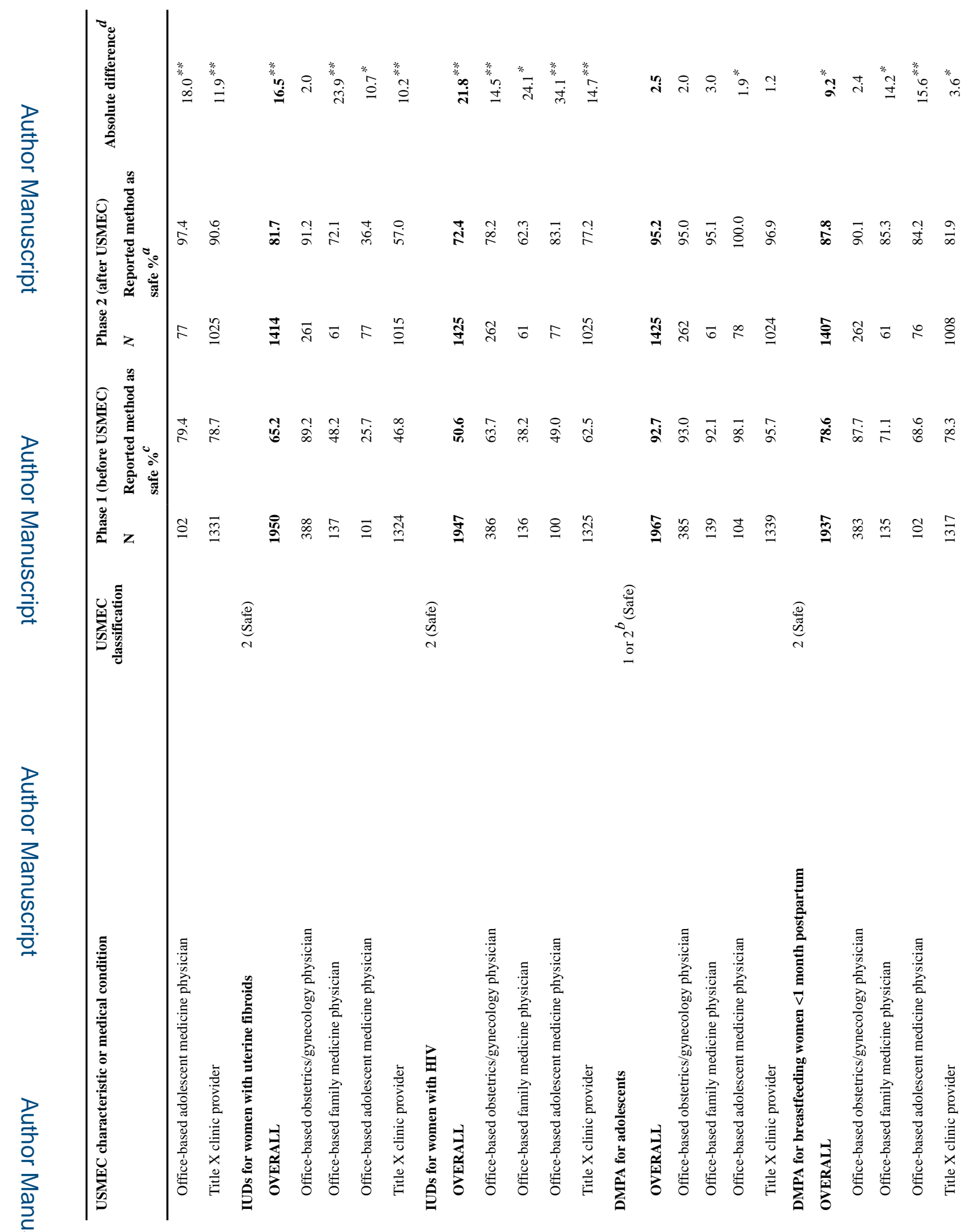




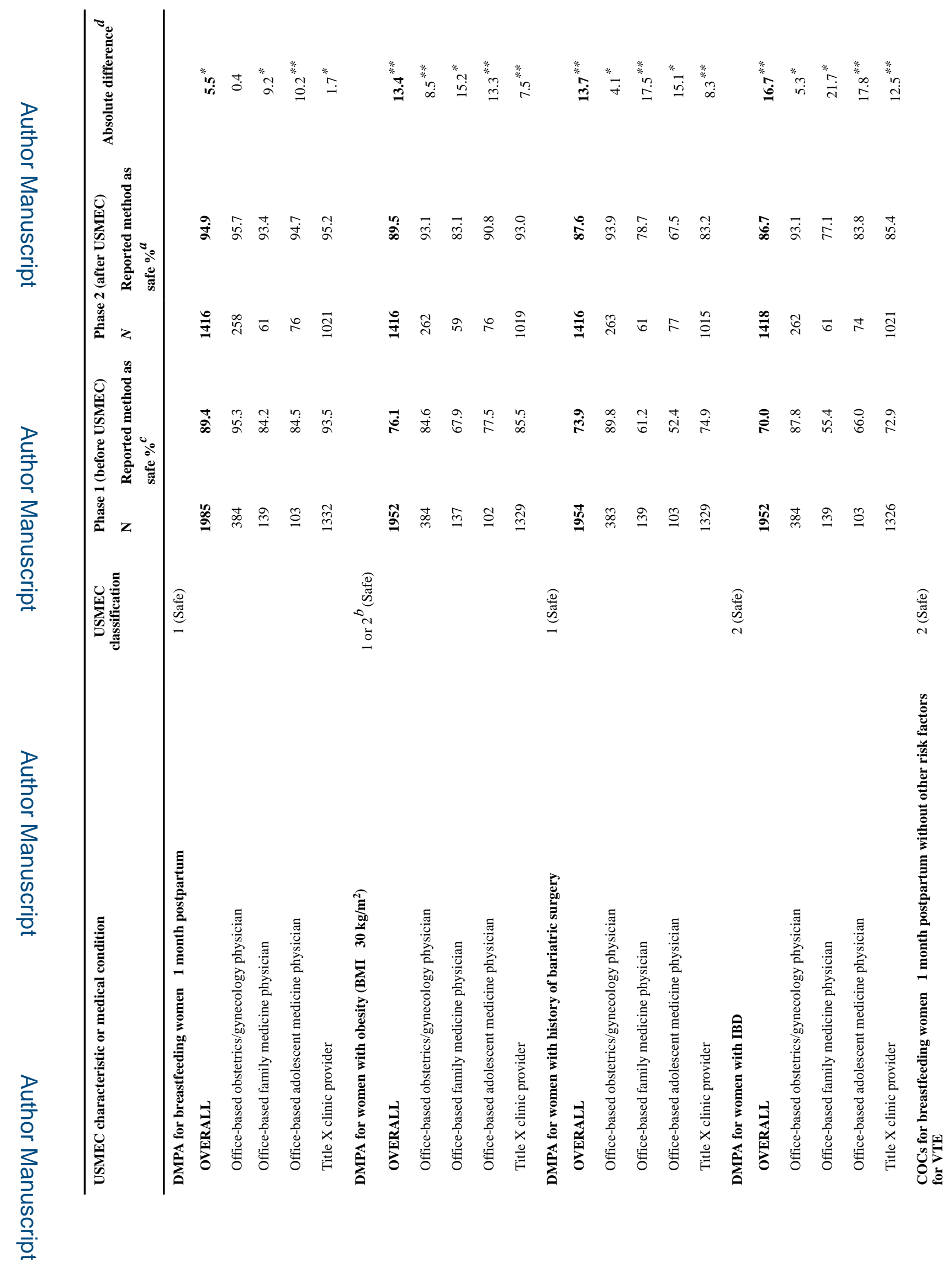

Contraception. Author manuscript; available in PMC 2020 July 22. 


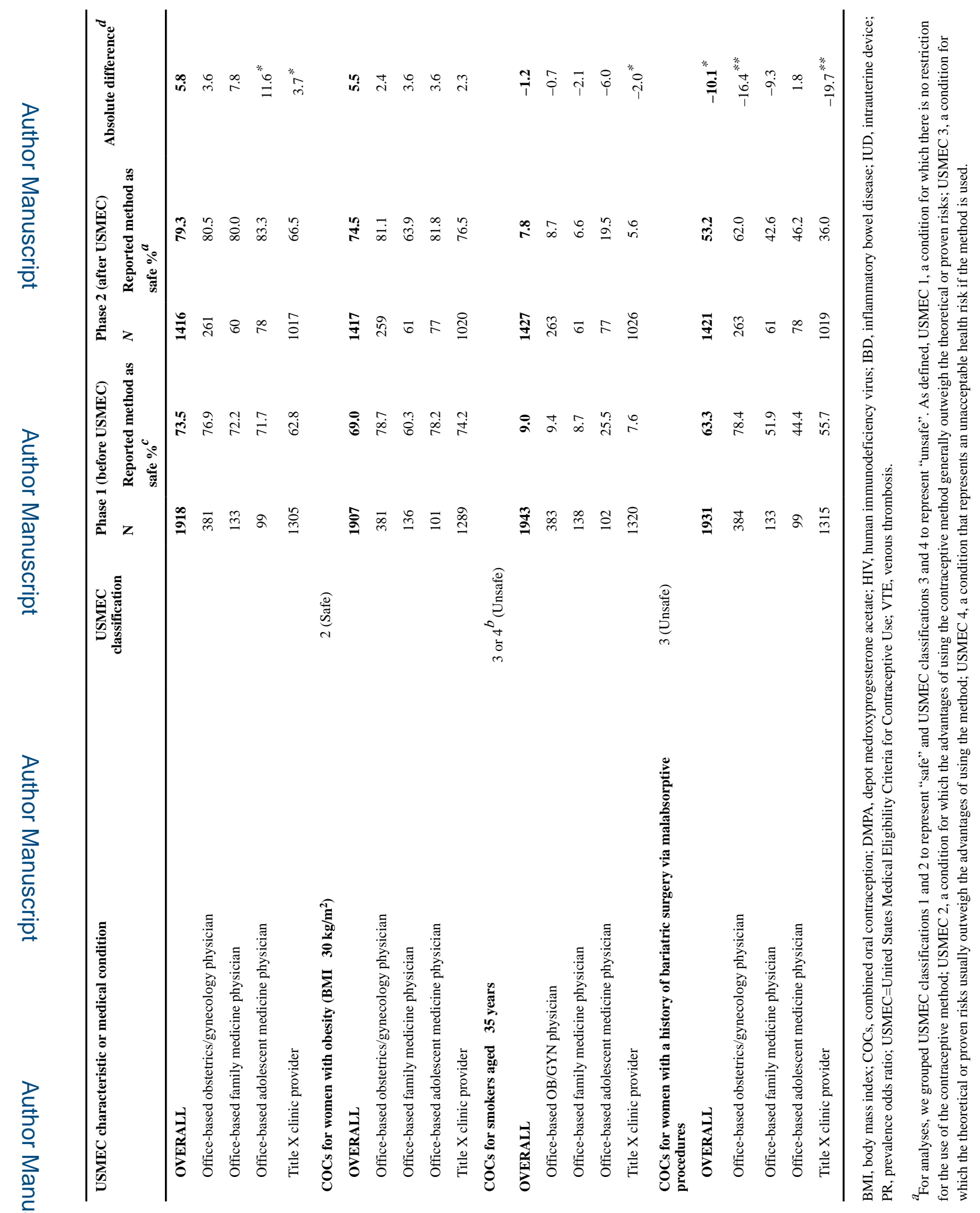

Contraception. Author manuscript; available in PMC 2020 July 22. 


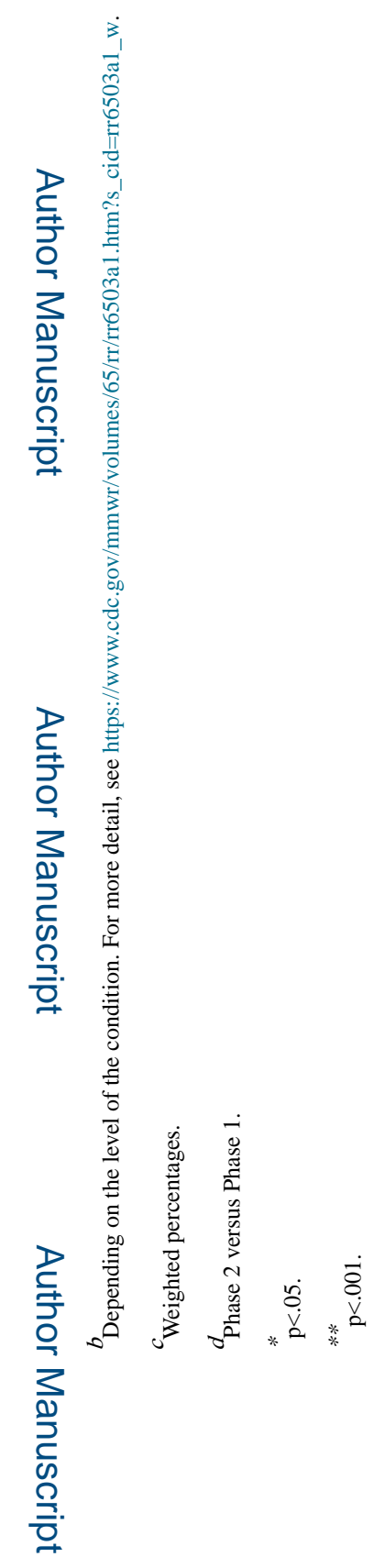

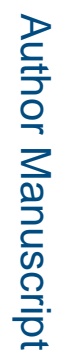




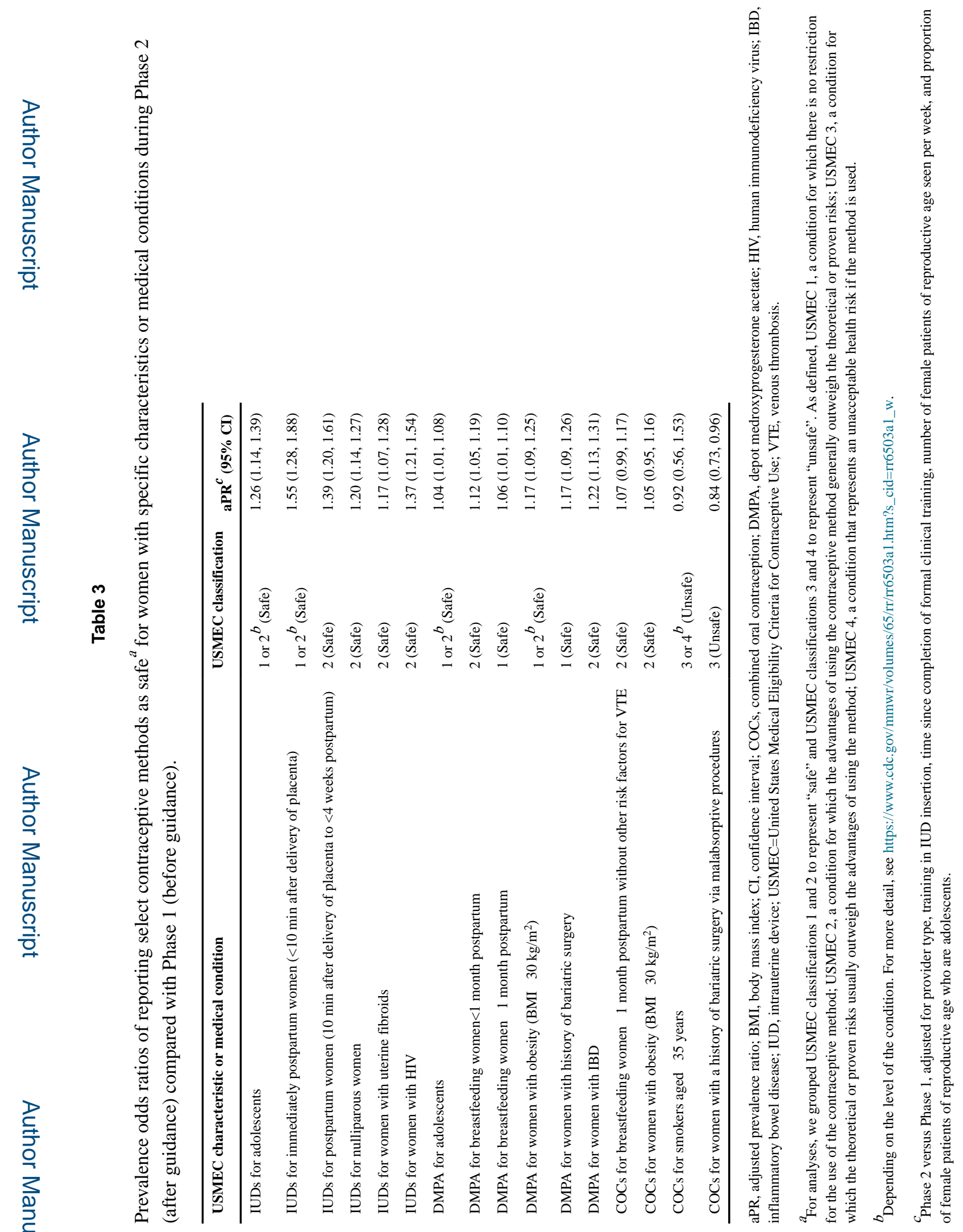

Contraception. Author manuscript; available in PMC 2020 July 22. 


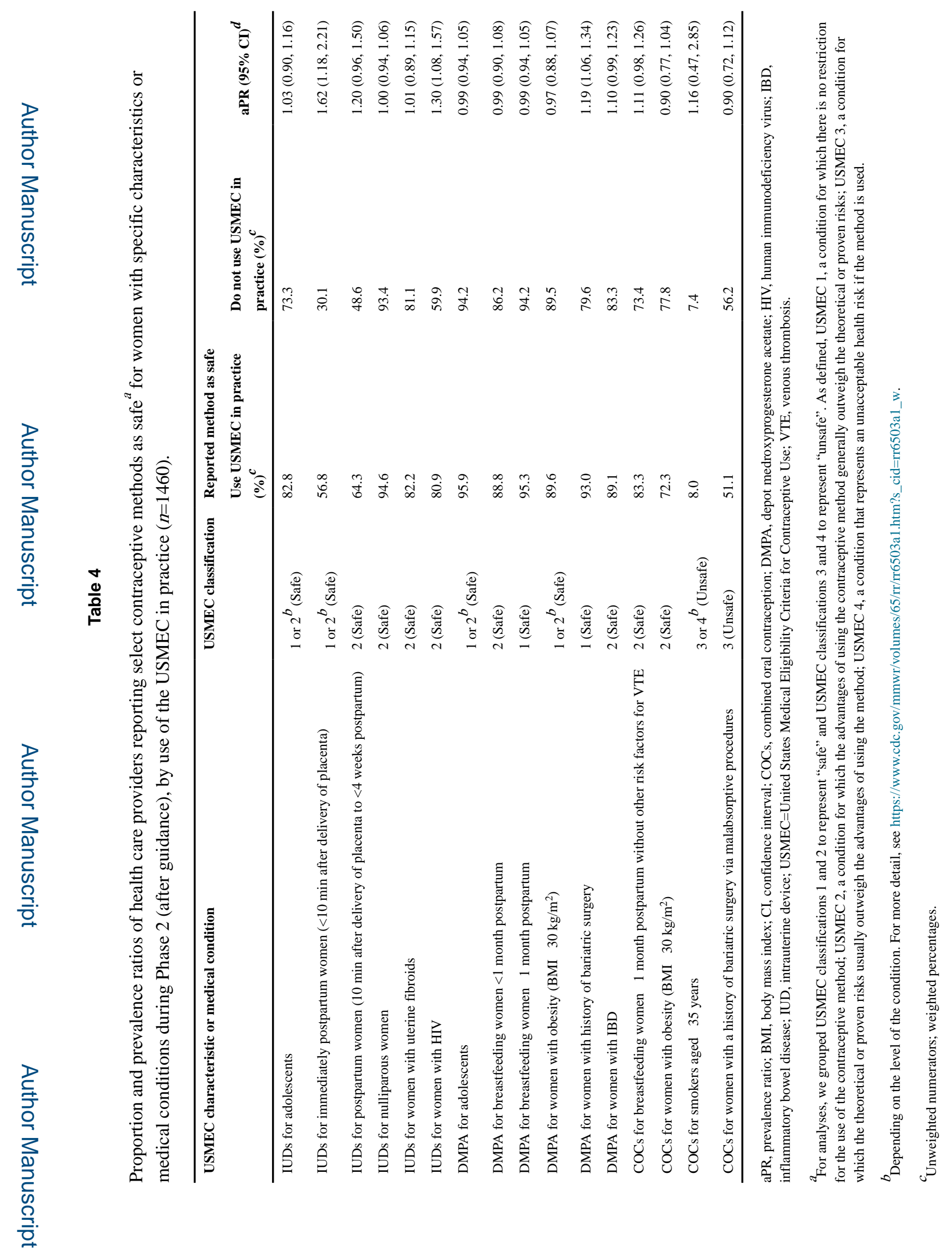

Contraception. Author manuscript; available in PMC 2020 July 22. 


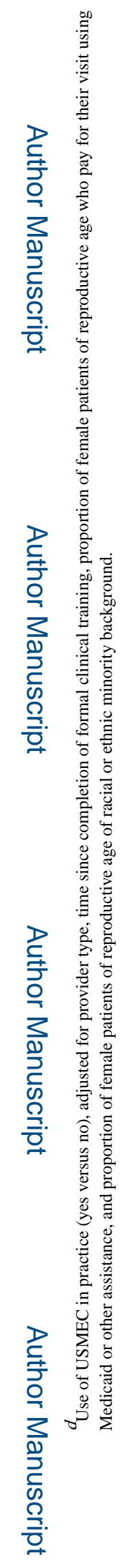

